\title{
Avaliação da capacidade funcional de idosos cadastrados na Estratégia de Saúde da Família na comunidade do Pontal da Barra, Maceió-AL
}

\author{
Emanuella Pinheiro de Farias Bispo ${ }^{a}$, Michelle Carolina Garcia da Rocha ${ }^{b}$, \\ Maria de Fátima Machado Reys Rocha ${ }^{\mathrm{c}}$ \\ aTerapeuta Ocupacional, Graduada, Residente em Saúde da Família, \\ Universidade Estadual de Ciências de Saúde de Alagoas - UNCISAL, Maceió, AL, Brasil \\ ${ }^{b}$ Fonoaudióloga, Mestre em Ciências da Linguagem, Universidade Católica de Pernambuco - UNICAP, \\ Professora Assistente, Preceptora da Residência Multiprofissional em Saúde da Família, \\ Universidade Estadual de Ciências de Saúde de Alagoas - UNCISAL, Maceió, AL, Brasil \\ 'Fisioterapeuta, Professora, Faculdade de Administração de Maceió - FAA, \\ Preceptora da Residência Multiprofissional em Saúde da Família, \\ Universidade Estadual de Ciências de Saúde de Alagoas - UNCISAL, Maceió, AL, Brasil
}

\begin{abstract}
Resumo: Com o crescente aumento do número de idosos, cresce, também, a necessidade de utilização de instrumentos de avaliação funcional. A capacidade funcional surge como um valor ideal para que o idoso possa viver independente, sendo essa a capacidade de o indivíduo realizar suas atividades físicas e mentais necessárias para manutenção de suas atividades básicas cotidianas. O estudo prospectivo, de corte transversal, teve por objetivo avaliar a capacidade funcional de idosos cadastrados na estratégia de saúde da família na comunidade do Pontal da Barra e a influência do gênero e da idade. A avaliação da capacidade funcional foi realizada a partir da aplicação da HAQ-Stanford Health Assesssment Questionnaire, com uma amostra de $\mathrm{n}=95$ idosos. Os resultados demonstraram que, na avaliação do gênero, constatou-se $n=43$ idosos do gênero masculino (45\%) e $n=52$, do gênero feminino (55\%), sendo utilizado, para análise de variância, o teste Kruskal-Wallis, e o pós-teste com $\mathrm{o}$ T-student, com valor de $\mathrm{p}=0,01$. Quanto à idade, dividiu-se em três grupos: de 60 a 70 anos, $\mathrm{n}=47$ idosos (50\%); 71 a 80 anos, $\mathrm{n}=39$ idosos (41\%), e 81 a 90 anos, $\mathrm{n}=9$ idosos (9\%). Cerca de $89 \%$ dos escores do HAQ obtiveram resultados entre os valores 0 e 2 , ou seja, os resultados demonstraram que a maior parte dos idosos foi classificada como "sem incapacidade" e bem abaixo da média de máxima incapacidade. Conclui-se que a maioria dos idosos apresentou um nível de capacidade funcional mais próximo do "sem incapacidade", sendo, em sua maior parte, idosos capazes de realizar de forma independente suas atividades cotidianas.
\end{abstract}

Palavras-chave: Programa Saúde da Família, Atividades Cotidianas, Idoso, Saúde do Idoso.

\section{Assessment of functional capacity of the elderly in registered Family Health Strategy in the community of 'Pontal da Barra', Maceio, State of Alagoas}

\begin{abstract}
As the amount of elderly people increases, so does the need for the use of functional assessment instruments to evaluate them. Functional capacity appears as an optimum value for the elderly to live independently, and this is the person's ability to perform the physical and mental activities required for the maintenance of their basic activities of daily living. This prospective, cross-sectional study aimed to evaluate the functional capacity, as well as the influence of gender and age in the elderly registered in the family health strategy in the community of 'Pontal da Barra'. The functional capacity evaluation was performed through the application of
\end{abstract}

Autor para correspondência: Emanuella Pinheiro de Farias Bispo, Universidade Estadual de Ciências de Saúde de Alagoas - UNCISAL, Av. Gustavo Paiva, s/n, bloco 06, apto. 508, Cruz das Almas, CEP 57038-050, Maceió, AL, Brasil, e-mail: manuzinhato@ gmail.com Recebido: 14/4/2011; 1ª Revisão: 14/9/2011; Aceite final:18/12/2011. 
the HAQ- Health Assessment Questionnaire (Stanford) to a sample of 95 individuals, $n=95$. The results showed that the evaluation of gender found $n=43$ elderly males (45\%) and $n=52$ females (55\%), using ANOVA, Kruskal-Wallis, and T-student test, with $\mathrm{p}=0.01$. Regarding the age, the sample was divided in three groups: aged 60 to 70 years old, $n=47(50 \%)$; aged 71 to $80, n=39(41 \%)$; and 81 to $90, n=9(9 \%)$. In about $89 \%$ of the HAQ responses, the results obtained were of values between 0 and 2, that is, most of the senior citizens were classified as "no disability" and were well below the average maximum disability. It was possible to conclude that the majority of the elderly show a level of functional capacity close to the "no disability" and that most of them are able to perform daily activities independently.

Keywords: Family Health Program, Activity of Daily Living, Aged, Aging Health.

\section{Introdução}

O Ministério da Saúde (MS) assumiu, em 1994, como estratégia setorial para reorientar o modelo assistencial brasileiro, o Programa de Saúde da Família (PSF) (GIROTI; NUNES; RAMOS, 2008), que corresponde a uma reorganização da prática da atenção à saúde em novas bases e uma substituição do modelo tradicional vigente, aproximando as açóes de saúde da família e, com isso, melhorando a qualidade de vida dos brasileiros (SANTANA; CARMAGNANI, 2001).

Um dos olhares dentro dessa comunidade volta-se para a populaçáo idosa, que vem aumentando consideravelmente nas últimas décadas. Segundo Lacourt e Marini (2006), dentre as principais alteraçôes que surgem com o avanço da idade está o decréscimo da funçáo muscular, que afeta diretamente a capacidade de realizar tarefas do dia a dia, diminuindo a independência funcional e, desse modo, interferindo na qualidade de vida do idoso.

A saúde do idoso é uma política do Ministério da Saúde que objetiva, no âmbito do Sistema Único de Saúde-SUS, garantir atençáo integral à saúde da população idosa, enfatizando o envelhecimento saudável e ativo, e fortalecendo o protagonismo das pessoas idosas no Brasil (Portaria no 2.528, de 19 de outubro de 2006). São diretrizes importantes para a atenção integral à saúde do idoso: 1) promoção do envelhecimento ativo e saudável; 2) manutenção e reabilitação da capacidade funcional; 3) apoio ao desenvolvimento de cuidados informais (BRASIL, 2009).

Um dos grandes problemas do processo de envelhecimento humano está associado à perda da capacidade funcional, tornando o idoso total ou parcialmente dependente, e afetando sua autonomia e qualidade de vida. Assim, a capacidade funcional surge como um valor ideal para que o idoso possa viver de forma independente e autônoma, sendo capaz de realizar suas atividades físicas e mentais necessárias para manutenção de suas atividades básicas, como: tomar banho; vestir-se; realizar higiene pessoal; transferir-se; alimentar-se; manter a continência; preparar refeiçōes; ter o controle financeiro etc (ALENCAR et al., 2008).

Estudar incapacidade funcional em idosos é importante para entender como as pessoas vivem os anos seguintes de suas vidas, sendo o aumento da longevidade um aspecto importante a ser considerado atualmente (PARAHYBA et al., 2005). A incapacidade funcional define-se pela presença de dificuldade no desempenho de certos gestos e de certas atividades da vida cotidiana ou mesmo pela impossibilidade de desempenhá-las (ROSA et al., 2003).

Quando o idoso está comprometido funcionalmente, a família é afetada também, já que a incapacidade ocasiona maior vulnerabilidade e dependência na velhice, contribuindo para a diminuição do bem-estar e da qualidade de vida dos idosos (ALVES et al., 2007).

A avaliação funcional, preconizada pela Política Nacional de Saúde da Pessoa Idosa, é fundamental e determinará não só o comprometimento funcional, mas sua necessidade de auxilio. Pode ser compreendida como uma tentativa sistematizada de avaliar de forma objetiva os níveis nos quais uma pessoa está funcionando numa variedade de áreas, utilizando diferentes habilidades (BRASIL, 2009).

A avaliação funcional pode ser definida como uma tentativa de medir, de forma objetiva, os níveis nos quais uma pessoa é capaz de desempenhar atividades ou funçôes em diferentes áreas, utilizando-se de várias habilidades para o desempenho das tarefas da vida cotidiana, para a realização de interaçóes sociais, em suas atividades de lazer e em outros comportamentos requeridos em seu dia a dia (DUARTE; ANDRADE; 
LEBRÃO, 2007). Esses autores complementam: essa definição é baseada no conceito de função, que corresponde à capacidade do indivíduo para adaptar-se aos problemas de todos os dias, apesar de possuir uma incapacidade física, mental e/ou social.

Avaliar significa analisar o processo de realização de atividades significativas em contextos de desempenho, em condiçôes ambientais nas quais o sujeito vive, no cotidiano, de modo a contribuir para que ele tenha melhor qualidade de vida, não ignorando os componentes da função ocupacional, tais como resistência, força e capacidade de solucionar problemas (MELLO et al., 2004; LAW, 2005).

$\mathrm{O}$ processo de avaliação envolve a coleta de dados e sua interpretaçáo. O terapeuta precisa ter habilidades e instrumentos para extrair os dados relevantes, interpretando-os completamente (SAURON; OLIVEIRA, 2003). A utilização de um instrumento de avaliação é muito importante e necessita de um treinamento, de disponibilidade de um manual e de facilidade de administração e interpretação; é necessário, ainda, o instrumento de avaliação ser validado e confiável (LAW, 2005).

Na reabilitação, a unidade de análise é o indivíduo e sua relação em seu meio ambiente, pois o objetivo é a melhoria das habilidades para a função, de forma mais independente possível, no seu ambiente natural. São necessários, portanto, instrumentos de avaliação que possibilitem averiguar as atividades e os ambientes (SAURON; OLIVEIRA, 2003).

$O$ interesse em realizar esta pesquisa na comunidade do Pontal da Barra, no município de Maceió-AL, surgiu da existência de um grande número de indivíduos idosos cadastrados na Estratégia de Saúde da Família, possivelmente com suas capacidades funcionais alteradas. Nesse sentido, a pesquisa teve por objetivo avaliar a capacidade funcional de idosos cadastrados na estratégia de saúde da família nessa comunidade.

\section{Materiais e métodos}

O estudo do tipo prospectivo e de corte transversal foi realizado na comunidade do Pontal da Barra (área IV), no município de Maceió-AL.

\subsection{Amostra e critérios de inclusão}

Foram inclusos na pesquisa todos os usuários idosos (a partir de 60 anos), cadastrados na Estratégia de Saúde da Família dessa comunidade, totalizando $\mathrm{n}=247$ idosos. Após o cálculo do tamanho da amostra, $\mathrm{n}=95$ sujeitos passaram a ser uma amostra representativa para a pesquisa. $\mathrm{O}$ cálculo do tamanho da amostra foi realizado com o teste quadrado. Os dados são expressos em média e desvio padrão, com nível de significância de 5\% ( $\mathrm{p}<0,05)$.

Foram excluídos da pesquisa os idosos que apresentaram doenças degenerativas, em coma e/ou com comprometimento cognitivo, da audição e de comunicação (linguagem). Para mobilização dos idosos para a pesquisa, foram utilizadas as fichas 'A' (ficha de cadastro) e valeu-se do auxílio dos agentes comunitários de saúde (ACS), que forneceram a lista de idosos de suas respectivas microáreas.

\subsection{Instrumento da coleta de dados - Stanford Health Assessment Questionnaire (HAQ)}

O HAQ (ARAÚJO et al., 2007) é um instrumento já validado no Brasil, que pode ser utilizado com a população adulta e idosa. É mais indicado para casos em que há comprometimento motor (MELLO; MANCINI, 2007). Como todo instrumento, o HAQ tem limitaçôes e, pelo fato de ser usado geralmente, não captura a inabilidade associada com a disfunção sensorial do órgão ou a disfunção psiquiátrica, e não mede diretamente a satisfação do paciente ou o trabalho em rede social. Contudo, tais variáveis ou outras do interesse do usuário podem prontamente ser adicionadas (BRUCE; FRIES, 2003).

O HAQ é um questionário autoadministrável, composto de 20 questóes, que mede a capacidade funcional após procedimentos terapêuticos, assim como fornece prognósticos em pacientes idosos ou cronicamente doentes. Essas questóes estáo agrupadas em oito categorias, que avaliam o nível de dificuldade que cada paciente apresenta para realizar determinadas atividades, assim como a necessidade de assistência para realizá-las. Para cada item, há uma escala da dificuldade em quatro níveis, em que é marcado de 0 (zero) a 3 (três): normal, nenhuma dificuldade, valor 0 ; alguma dificuldade, valor 1 ; muita dificuldade, valor 2 , e incapaz de fazer, valor 3. Pode ser facilmente aplicado em apenas dez minutos (BRANDĀO; FERRAZ; ZERBINI, 1997; FORTES et al., 2008).

A variável primária correspondeu à capacidade funcional (valor do HAQ), que corresponde à possibilidade de um indivíduo funcionar normalmente, sem comprometimento das habilidades comportamentais ou motoras, ou alterações das funçôes sociais. As variáveis secundárias exploradas foram idade e gênero. 


\subsection{Procedimentos de coleta e análise de dados}

A pesquisa foi realizada no domicílio de casa idoso, sendo uma microárea por vez. Em seguida, foi aplicado o HAQ, sendo avaliada a capacidade funcional de cada idoso. Depois de coletados e apurados todos os dados da pesquisa, foi construída uma tabela no programa Excel 2007, com os resultados dos escores e os valores parciais de cada grupo do questionário (8 grupos ao total), sendo então realizada uma análise quantitativa, com auxílio do programa de estatística BioEstat. Além disso, foram criados gráficos explicativos no programa Word 2007.

\subsection{Aspectos éticos}

Conforme deliberação em plenária ordinária do CEP/UNCISAL, ocorrida no dia 15/set./2009, foi de consenso a aprovaçáo deste trabalho sob o protocolo no 1193 . Após a aprovação, a pesquisa foi iniciada. Antes da aplicação do questionário, foi apresentado a cada idoso e/ou acompanhante o Termo de Consentimento Livre e Esclarecido. Este foi lido por um dos pesquisadores ou de preferência pelo usuário selecionado, e todas as dúvidas pertinentes ao modelo do consentimento foram retiradas pela pesquisadora responsável, conforme a resolução 196/96 do CNS.

\section{Resultados e discussão}

A amostra contou com um total de $\mathrm{n}=95$ idosos, conforme explicitado anteriormente. Para confirmar se os dados apresentavam distribuiçáo normal, foi realizado teste de normalidade (Lilefors), cujo valor de p foi igual a 0,09 , ou seja, maior que 0,05 , apresentando distribuição normal das variáveis. Assim, foram utilizados testes paramétricos, já que as variáveis são quantitativas e, dessa forma, possuem distribuição normal.

Em uma primeira etapa, avaliou-se o gênero dos idosos pesquisados, constatando-se $\mathrm{n}=43$ idosos do gênero masculino (45\%) e n $=52$, do gênero feminino (55\%). Foi utilizado, para análise de variância, o teste Kruskal-Wallis, pelo qual foi detectada diferença entre os grupos. Assim, foi realizado o pós-teste com o T-student, em cujo resultado observou-se um valor de alfa de $\mathrm{p}=0,01$ (Tabela 1 ), ou seja, significativo, menor que 0,05 .

Em outro estudo (ROSA et al., 2003), observou-se que, em relação à variável quantitativa de gênero, além de uma predominância feminina, o p foi inferior a 0,001 . Por meio de uma avaliaçáo comparativa dos escores do HAQ entre os gêneros, observou-se que, em uma média aritmética, o gênero feminino possui uma média total do valor do $\operatorname{HAQ}(0,95)$ maior que o gênero masculino $(0,69)$. Esse fato pode ser justificado pela adoção de hábitos saudáveis pelas mulheres, enquanto os homens mantêm, principalmente, os vícios do álcool e do tabagismo. Segundo Rosa et al. (2003), o gênero está fortemente associado à ocorrência da dependência, sendo mais de duas vezes maior a chance para as mulheres em relação aos homens, diferenciando-se desta pesquisa.

Quanto à idade, os sujeitos foram divididos em três grupos: de 60 a 70 anos, $\mathrm{n}=47$ idosos $(50 \%)$; 71 a 80 anos, $\mathrm{n}=39$ idosos (41\%); 81 a 90 anos, $\mathrm{n}=9$ idosos (9\%). Foi utilizado, para análise de variância, o teste Kruskal-Wallis, sendo identificado um $\mathrm{p}$ superior a 0,05 , com valor de $\mathrm{p}=0,13$ (Tabela 2), ou seja, não significativo.

Em outra pesquisa (FORTES et al., 2008), utilizando essa mesma variável quantitativa de idade e associando ao gênero, observou-se um p = 0,079, em uma populaçáo acima de 60 anos, porém com características clínicas diferenciadas.

Em uma terceira etapa, outra questão importante analisada foi em relaçấo aos resultados dos escores do questionário aplicado. Em $89 \%$ dos escores do $\mathrm{HAQ}$, foram obtidos resultados entre os valores 0 e 2 , ou seja, resultados que demonstram que a maior parte dos idosos foi classificada como sem incapacidade e bem abaixo da média de máxima incapacidade, fato que pode ser associado a uma perda da capacidade normal para a fase idosa, devido aos comprometimentos orgânicos naturais da idade.

Em seu estudo, Nakatani et al. (2003) traz que a idade, a acuidade visual diminuída, as doenças associadas, a depressão, o equilíbrio e a mobilidade

Tabela 1. Perfil da amostra quanto ao gênero.

\begin{tabular}{cl}
\hline Gênero & N \\
\hline Masculino* & 43 \\
Feminino* & 52 \\
\hline
\end{tabular}

$* p=0,01$

Tabela 2. Amostra participante da avaliação de capacidade funcional segundo a idade.

\begin{tabular}{cc}
\hline Idade (anos) & $\mathbf{N}$ \\
\hline $60-70$ & 47 \\
$71-80$ & 39 \\
$81-90$ & 9 \\
\hline
\end{tabular}


prejudicados estão estatisticamente associados à dependência dos idosos Segundo Lacourt e Marini (2006), o processo de envelhecimento vem acompanhado de diversas alterações, que vão causando danos aos diferentes sistemas do organismo. Já os valores de 2 a 3 (11\%) corresponderam a uma parcela pequena do total de idosos, o que demonstra, em um panorama geral, que os idosos deste estudo são capazes de realizar suas atividades cotidianas.

Outro estudo (SCHNEIDER; MARCOLIN; DALACORTE, 2008) traz que indivíduos idosos com limitações funcionais, principalmente, apresentam maiores restriçôes às suas Atividades da Vida Diária - AVD, tornando-os menos independentes.

Outro ponto importante que este estudo traz é um grande número de idosos com escore 0 , ou seja, de sem incapacidade, que totaliza $\mathrm{n}=26$ idosos. Tal dado pode se justificar pelo fato de serem idosos com uma boa qualidade de vida. É importante comentar que saúde não é apenas uma questão de assistência médica e de acesso a medicamentos, mas sim a promoção de "estilos de vida saudáveis", encarada pelo sistema de saúde como uma ação estratégica (BRASIL, 2009).

Em contrapartida, o valor 3 (máxima incapacidade) foi identificado em apenas $n=6$ idosos, dos $n=96$ entrevistados, o que demonstra um número pequeno comparado ao número total de idosos.

Como o HAQ é divido em oito grupos, em que se avaliam os níveis de dificuldade em realizar determinadas atividades de acordo com as funçóes, analisaram-se os resultados de cada grupo individualmente, comparando os dados mais pertinentes.

Assim, de acordo com a Figura 1, alguns grupos de questóes tiveram valor 0 (sem incapacidade) individualmente. Os grupos com maior número de idosos que tiveram valor 0 foram os grupos $3(18 \%)$, $1(16 \%), 2$ (15\%) e 5 (14\%). O grupo 3 corresponde ao conjunto de questóes: Cortar pedaços de carne / Levar à boca um copo ou uma xicara cheia de água / Abrir um pacote de leite comum; o grupo 1 corresponde a: Vestir-se (abotoar a camisa e amarrar os sapatos) / Lavar a cabeça e os cabelos; o grupo 2 corresponde a: Levantar-se de maneira reta de uma cadeira com encosto e sem braços / Deitar-se e levantar-se da cama; e o grupo 5: Lavar e secar o corpo após o banho / Tomar banho de chuveiro / Sentar-se e levantar-se de um vaso sanitário.

As questóes relacionadas a esses grupos são referentes a atividades que necessitam de uma coordenação motora fina a uma coordenação motora grossa, além de uma atividade ativa dos membros superiores, da coluna e da cadeia muscular posterior. Tal quadro ocorre talvez pelo fato de serem idosos ativos - pescadores e rendeiras, na sua maioria que fazem sua atividade laboral há muitos anos, possuindo, assim, um preparo físico para realizar tarefas que proporcionam uma descarga maior nessas regiōes corporais.

Alves et al. (2007) pontuam que a manutenção da capacidade funcional pode ter implicaçóes para a qualidade de vida dos idosos, pois está diretamente relacionada com a forma pela qual o indivíduo se mantém na comunidade, desfrutando a sua independência até as idades mais avançadas. Assim, a detecção precoce e a avaliação periódica dos parâmetros funcionais fazem-se necessárias, a fim de se manterem pelo maior tempo possível a autonomia e o bem-estar do indivíduo (SCHNEIDER; MARCOLIN; DALACORTE, 2008).

A Figura 2 dispóe que alguns grupos tiveram valor 3 (máxima incapacidade). Nos grupos 7 (21\%) e 8 (20\%), o valor 3 foi alto em relaçáo aos outros grupos. O grupo 7 corresponde às questóes: Segurar-se em pé no ônibus ou trem / Abrir potes ou vidros de conservas que já tenham sido abertos / Abrir e fechar torneiras; e o grupo 8: Fazer compras perto de onde mora / Entrar e sair do ônibus / Varrer e usar o rodo para o chão.

Pode-se observar, com a Figura 2, que as questóes que mais apresentaram dificuldades de realização foram as que necessitavam de movimentos de punho, principalmente, os desvios ulnar e radial (grupo 7). Também é relevante citar a questão do deambular

Grupos (G) do HAQ com valor 0 (sem dificuldade)

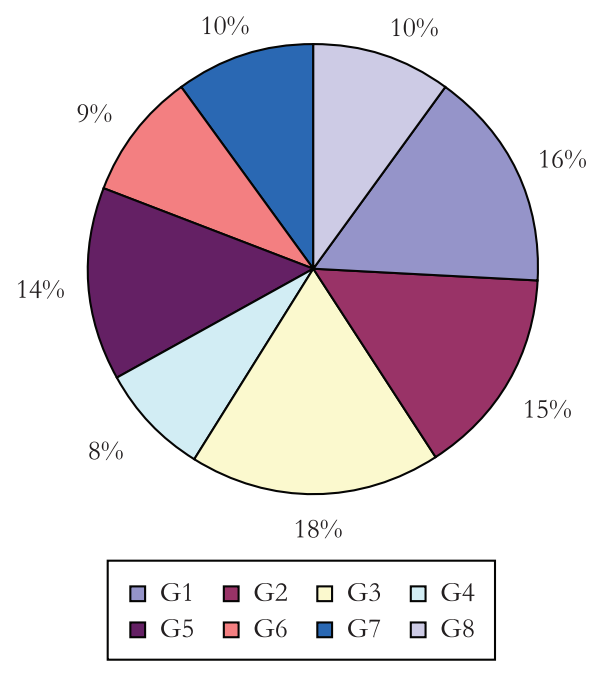

Figura 1. Grupos (G) do HAQ com valor 0 (sem dificuldade). 
Grupos (G) com valor 3 (máxima incapacidade)

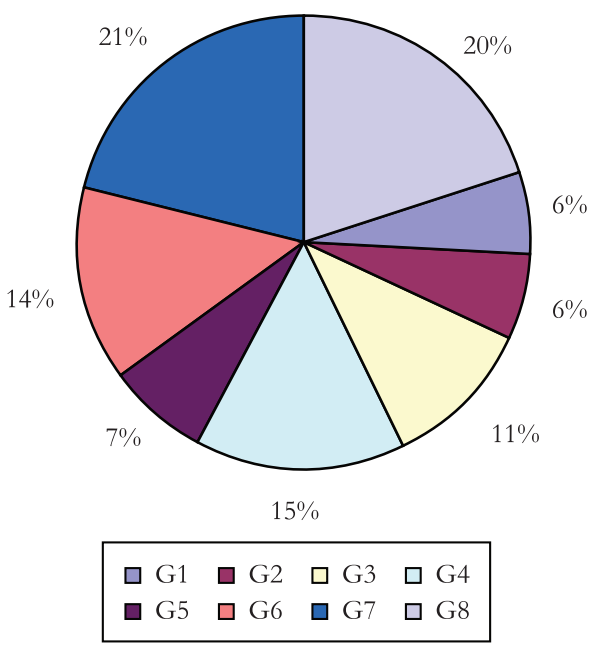

Figura 2. Grupos (G) com valor 3 (Máxima incapacidade).

e de fazer atividades que necessitem do esforço da regiáo da coluna lombar e torácica, que também são regióes desgastadas por esses idosos, pescadores e rendeiras, em sua maioria.

Rosa et al. (2003, p. 47), afirmam que:

[...] a manutenção da capacidade funcional pode ter importantes implicaçôes para a qualidade de vida dos idosos, por estar relacionada com a capacidade de ocupar-se com o trabalho até idades mais avançadas e/ou com atividades agradáveis. Parece, portanto, bastante relevante planejar programas específicos de intervenção para a eliminaçáo de certos fatores de risco relacionados com a incapacidade funcional.

Na comunidade do Pontal da Barra, comunidade ribeirinha, os idosos são bastante ativos, participativos em associaçôes, grupos educativos (grupo de homens e de idosos) e de alongamento. O estabelecimento de açôes preventivas é possibilitado pelo manejo de açôes em atividades sociais, promovendo a ação de grupos, descobrindo as potencialidades, trabalhando a vulnerabilidade e estimulando uma vida saudável e associativa (ROSA et al., 2003; BRASIL, 2009).

\section{Conclusão}

Os resultados deste trabalho permitem concluir que a maioria dos idosos participantes deste estudo apresentou um nível de capacidade funcional mais próxima do "sem incapacidade"; ou seja: eram, em sua maioria, idosos capazes de realizar de forma independente suas atividades cotidianas, sendo, assim, um grupo de idosos diferenciado. Destaca-se, a partir do presente estudo, a necessidade da investigação de alguns fatores determinantes que podem influenciar na capacidade funcional, como os fatores socioeconômicos, demográficos e relacionais, e a autopercepção de saúde. Acredita-se que, a partir da identificação do perfil dos idosos quanto ao seu nível de capacidade funcional, será possível elaborar estratégias e açôes direcionadas para esse público, principalmente no que diz respeito às questôes funcionais.

\section{Referências}

ALENCAR, M. C. B.; HENEMANN, L.; ROTHENBUHELER, R. A capacidade funcional de pacientes, e a fisioterapia em um programa de assistência domiciliar. Revista Fisioterapia em Movimento, v. 21, n. 1, p. 11-20, 2008.

ALVES, L. C. et al. A influência das doenças crônicas na capacidade funcional dos idosos do Município de São Paulo, Brasil. Cadernos de Saúde Pública, v. 23, n. 8, p. 1924-1930, 2007. PMid:17653410.

ARAÚJO, C. D. M. et al. Influência da hipertrofia mamária na capacidade funcional das mulheres. Revista Brasileira de Reumatologia, v. 47, n. 2, p. 91-96, 2007.

BRANDĀO, L.; FERRAZ, M. A.; ZERBINI, C. A. F. Avaliação da qualidade de vida na artrite reumatóide: revisão atualizada. Revista Brasileira de Reumatologia, v. 37, n. 5, p. 275-281, 1997.

BRASIL. Ministério da Saúde. Saúde do Idoso. Portal da Saúde, 2009. Disponível em: <http://portal.saude.gov.br/ portal/saude/visualizar_texto.cfm?idtxt=26466>. Acesso em: 28 nov. 2010.

BRUCE, B.; FRIES, J. F. The stanford health assessment questionnaire: a review of its history, issues, progress, and documentation. The Journal of Rheumatology, v. 30, n. 1, p. 167-78, 2003. Disponível em: <http://aramis.stanford. edu/downloads/JRheumatol_new\%20HAQ.pdf>.

DUARTE, Y. A. O.; ANDRADE, C. L A.; LEBRÃO, M L. O Índex de Katz na avaliação da funcionalidade dos idosos. Revista da Escola de Enfermagem - USP, v. 41, n. 2, p. 317-25, 2007. http://dx.doi.org/10.1590/ S0080-62342007000200021

FORTES, E. M. et al. Elevada morbimortalidade e reduzida taxa de diagnóstico de osteoporose em idosos com fratura de fêmur proximal na cidade de São Paulo. Arquivos Brasileiros de Endocrinologia \& Metabologia, v. 52, n. 7, p. 1106-1114, 2008.

GIROTI, S. K. O.; NUNES, E. F. P. A.; RAMOS, M. L. $\mathrm{R}$. As práticas das enfermeiras de uma unidade de saúde da família de Londrina, e a relação com as atribuiçóes do exercício profissional. Universidade Estadual de Londrina. Ciências Biológicas e da Saúde, v. 29, n. 1, p. 9-26, 2008. LACOURT, M. X.; MARINI, L. L. Decréscimo da função muscular decorrente do envelhecimento e a 
influencia na qualidade de vida do idoso: uma revisão de literatura. RBCEH - Revista Brasileira de Ciências do Envelhecimento Humano, v. 3, n. 1, p. 114-121, 2006.

LAW, M. Avaliando papéis e competência. In: TROMBLY, C. A.; R ADOMSKI, M. V. Terapia Ocupacional para disfunçôes físicas. 5. ed. São Paulo: Santos, 2005. p. 31-45.

MELLO, M. A. F. et al. Processo avaliativo em Terapia Ocupacional. In: CARLO, M. M. R. P.; LUZO, M. C. Terapia Ocupacional - reabilitação física e contextos hospitalares. São Paulo: Roca, 2004. p. 74-98

MELLO, M. A. F.; MANCINI, M. C. Métodos e técnicas de avaliação nas áreas de desempenho ocupacional, seção: avaliação das atividades de vida diária e controle domiciliar. In: CAVALCANTI, A.; GALVÃO, C. Terapia Ocupacional fundamentação e prática. Rio de Janeiro: Guanabara Koogan, 2007. p. 49-52.

NAKATANI, A. Y. K. et al. Perfil sócio-demográfico e avaliação funcional de idosos atendidos por uma equipe de saúde da família na periferia de Goiânia. Revista da Sociedade Brasileira de Clínica Média, v. 5, n. 1, p. 131-136, 2003.
PARAHYBA, M. I.; VERAS, R.; MELZER, D. Incapacidade funcional entre as mulheres idosas no Brasil. Revista de Saúde Pública, v. 39, n. 3, p. 383-391, 2005. http://dx.doi.org/10.1590/S0034-89102005000300008 ROSA, T. E. C. et al. Fatores determinantes da capacidade funcional entre idosos. Revista de Saúde Pública, v. 37, n. 1, p. 40-8, 2003. http://dx.doi.org/10.1590/ S0034-89102003000100008

SANTANA, M. L.; CARMAGNANI, M. I. Programa saúde da família no Brasil: um enfoque sobre seus pressupostos básicos, operacionalização e vantagens. Revista Saúde e Sociedade, v. 10, n. 1, p. 33-53, 2001.

SAURON, F. N.; OLIVEIR A, M. C. Avaliação em Terapia Ocupacional: aspectos práticos. In: TEIXEIRA E. et al. Terapia Ocupacional na reabilitação física. São Paulo: Roca, 2003. p. 221-236.

SCHNEIDER, R. H.; MARCOLIN, D.; DALACORTE, R. R. Avaliação funcional de idosos. Revista Scientia Medica, v. 18, n. 1, p. 4-9, 2008.

\section{Contribuição dos Autores}

Emanuella Pinheiro de Farias Bispo: Concepção do texto manuscrito; Organização de fontes e análises de dados; redaçấo do texto. Michelle Carolina Garcia da Rocha e Maria de Fátima Machado Reys Rocha: Revisão do texto. 